\title{
Fixed point theorems under some contractive conditions in partial Hausdorff metric spaces
}

\author{
Hacer Bozkurt \\ Department of Mathematics, Batman University, Batman, Turkey
}

Received: 7 April 2019, Accepted: 21 June 2019

Published online: 29 September 2019.

\begin{abstract}
In this paper, we investigate some set-valued contraction mappings in partial Hausdorff metric spaces and prove the existence of fixed point of this set-valued mappings in partial Hausdorff metric spaces. We also give an example as support of our results.
\end{abstract}

Keywords: Fixed point, partial metric space, partial Hausdorff metric, multi-valued mapping.

\section{Introduction}

In 1920, Banach introduced and proved the famous Banach Contraction Principle. The fixed point theory in metric spaces is an important part of functional analysis since that day and many researchers have introduced new contraction mappings and new fixed point theorems. Matthews introduced the notion of a partial metric space in [1]. He extended Banach contraction principle in the setting of complete partial metric space in the same study. After, further studies have been made by many authors ([4],[11],[12], [9], [8], [2], [3] and [13]). In [5], they proved the some generalized versions of the fixed point theorem of Matthews and established a homotopy results. Based on the partial metric on a set $X$, in [7], they presented a notion of partial Hausdorff metric on the $\Omega_{C p}(X)$. Besides, in [7], they studied of fixed point theorem for multi-valued mappings on a partial metric space using the partial Hausdorff metric and generalized Nadler's fixed point theorem. Further, in [6], he introduced the notion of Banach G-contractions. Then many authors extended the Banach G-contraction different from Jachymaski [15], [16], [17] and [18].

The aim of this paper is to prove some fixed point theorems for set-valued mappings in Hausdorff metric spaces. Throughout this paper, $\Omega_{C}^{p}(X)$ is the family of all nonempty closed and bounded subsets of a partial metric space $(X, p)$.

Firstly, the following definition of partial metric space and some related results that are will be needed in the progression is given by Matthews ([1]).

Definition 1. [1] Let $X$ be a nonempty set. A mapping $p: X \times X \rightarrow \mathbb{R}^{+}$is a partial metric on $X$, if for all $x, y, z \in X$. We have

(p1) $p(x, x)=p(y, y)=p(x, y)$ if and only if $x=y$,

(p2) $p(x, x) \leq p(x, y)$,

(p3) $p(x, y)=p(y, x)$,

(p4) $p(x, z) \leq p(x, y)+p(y, z)-p(y, y)$.

The pair $(X, p)$ is then called a partial metric space. If $p(x, y)=0$, then $p 1)$ and $p 2)$ imply that $x=y$. But the reverse does not satisfy always. 
Example 1. [1] Let $X=\{[a, b]: a, b \in \mathbb{R}, a \leq b\}$, that is, $X=\Omega_{C}(\mathbb{R})$ and define a function $p: X \times X \rightarrow \mathbb{R}^{+}$is defined as $p(x, y)=p([a, b],[c, d])=\max \{b, d\}-\min \{a, c\}$, then $(X, p)$ is a partial metric space.

[1] Every metric space is a partial metric space. Each partial metric $p$ on $X$ generates a $T_{0}$ topology $\tau_{p}$ on $X$ with as a base the family of the open $p$-balls $\left\{B_{p}(x, \varepsilon): x \in X, \varepsilon>0\right\}$, where $B_{p}(x, \varepsilon)=\{y \in X: p(x, y)<p(x, x)+\varepsilon\}$.

Definition 2. [1] Let $(X, p)$ be a partial metric space. Then:

(a) A sequence $\left(x_{n}\right)$ in $(X, p)$ converges to a point $x \in X$ with respect to $\tau_{p}$ if and only if $p(x, x)=\lim _{n \rightarrow \infty} p\left(x, x_{n}\right)$.

(b) A sequence $\left(x_{n}\right)$ in $(X, p)$ is called Cauchy sequence if there exists and is finite $\lim _{n, m \rightarrow \infty} p\left(x_{n}, x_{m}\right)$.

(c) A partial metric space $(X, p)$ is called a complete partial metric space if every Cauchy sequence $\left\{x_{n}\right\}$ in $X$ converges with respect to $\tau_{p}$ to a point $x \in X$.

Remark. [1] Let $(X, p)$ be a partial metric space. Then the function $d_{p}: X \times X \rightarrow[0, \infty)$ defined by

$$
d_{p}(x, y)=2 p(x, y)-p(x, x)-p(y, y)
$$

is a metric on $X$. Let $(X, p)$ be a partial metric space, a sequence $\left\{x_{n}\right\}$ in $\left(X, d_{p}\right)$ is said to be convergent to a point $x \in X$ if and only if

$$
p(x, x)=\lim _{n \rightarrow \infty} p\left(x, x_{n}\right)=\lim _{n, m \rightarrow \infty} p\left(x_{n}, x_{m}\right) .
$$

Lemma 1.[7] Let $(X, p)$ be a partial metric space. Then:

(a) A sequence $\left(x_{n}\right)$ in $X$ is a Cauchy with respect to $p$ if and only if it is Cauchy with respect to $d_{p}$.

(b) A partial metric space $(X, p)$ is complete if and only if the metric space $\left(X, d_{p}\right)$ is complete.

Now, let us give the definition of partial Hausdorff metric space and reletad results. First, we remember and state the definition of Hausdorff metric for metric spaces.

Let $(X, d)$ be a metric space and $\Omega_{C}(X)$ denotes the collection of all nonempty closed and bounded subsets of $X$. For $A, B \in \Omega_{C}(X)$, define

$$
H(A, B)=\max \left\{\sup _{a \in A} d(a, B), \sup _{b \in B} d(b, A)\right\}
$$

where $d(x, A)=\inf \{d(x, a): a \in A\}$ is the distance of a point $x$ to the set $A$. We know that $H$ is a metric on $\Omega_{C}(X)$, called the Hausdorff metric induced by the metric $d$.

[7] In a partial metric space closedness is taken from $\left(X, \tau_{p}\right)$ and boundedness is given as follows: $A$ is a bounded subset in $(X, p)$ if there exist $x_{0} \in X$ and $M \geq 0$ such that for all $a \in A$, we have $a \in B_{p}\left(x_{0}, M\right)$, that is, $p\left(x_{0}, a\right)<p(a, a)+M$. Again in [7] following nations are defined. For $A, B \in \Omega_{C}^{p}(X)$ and $x \in X$,

$$
p(x, A)=\inf \{p(x, a), a \in A\} \text { and } \delta_{p}(A, B)=\sup \{p(a, B): a \in A\} .
$$

From here for the functions $\delta_{p}: \Omega_{C}^{p}(X) \times \Omega_{C}^{p}(X) \rightarrow \mathbb{R}^{+}$and $H_{p}: \Omega_{C}^{p}(X) \times \Omega_{C}^{p}(X) \rightarrow \mathbb{R}^{+}$, we have the following

$$
H_{p}(A, B)=\max \left\{\delta_{p}(A, B), \delta_{p}(B, A)\right\} .
$$

Remark. [5] Let $(X, p)$ be a partial metric space and $A$ be any nonempty subset of $X$, then

$$
a \in \bar{A} \text { if and only if } p(a, A)=p(a, a) .
$$


Proposition 1. [7] Let $\delta_{p}: \Omega_{C}^{p}(X) \times \Omega_{C}^{p}(X) \rightarrow \mathbb{R}^{+}$. For all $A, B, C \in \Omega_{C}^{p}(X)$, we have the following:

(i) $\delta_{p}(A, A)=\sup \{p(a, a): a \in A\}$,

(ii) $\delta_{p}(A, A) \leq \delta_{p}(A, B)$,

(iii) $\delta_{p}(A, B)=0$ implies that $A \subseteq B$,

(iv) $\delta_{p}(A, B) \leq \delta_{p}(A, C)+\delta_{p}(C, B)-\inf _{c \in C} p(c, c)$.

Proposition 2. [7] Let $(X, p)$ be a partial metric space. For any $A, B, C \in \Omega_{C}^{p}(X)$, we have

(1) $H_{p}(A, A) \leq H_{p}(A, B)$,

(2) $H_{p}(A, B)=H_{p}(A, B)$,

(3) $H_{p}(A, B) \leq H_{p}(A, C)+H_{p}(C, B)-\inf _{c \in C} p(c, c)$.

We know that a multi-valued mapping $T: X \rightarrow \Omega_{C}(X)$ is said to be contraction if

$$
H(T x, T y) \leq k d(x, y)
$$

for all $x, y \in X$ and for some $k \in[0,1)$. After above definition Nadler (in [14]) was proved the following theorem.

Theorem 1. [14] Let $(X, d)$ be a complete metric space and $T: X \rightarrow \Omega_{C}(X)$ be a contraction mapping. Then, there exists $x \in X$ such that $x \in T x$.

Lemma 2. [7] Let $(X, p)$ be a partial metric space, $A, B \in \Omega_{C}^{p}(X)$ and $h>1$. For any $a \in A$, there exists $b=b(a) \in B$ such that

$$
p(a, b) \leq h H_{p}(A, B) .
$$

Theorem 2. [7] Let $(X, p)$ be a complete partial metric space. If $T: X \rightarrow \Omega_{C}(X)$ is a multi-valued mapping such that for all $x, y \in X$, we have

$$
H_{p}(T x, T y) \leq k p(x, y)
$$

where $k \in(0,1)$. Then $T$ has a fixed point.

\section{Main results}

In this section, we give some fixed point results for multi-valued mappings on a complete partial metric space.

Theorem 3. Let $(X, p)$ be a complete partial metric space and let $T: X \rightarrow \Omega_{C}^{p}(X)$ be a set-valued map such that

$$
H_{p}(T x, T y) \leq \alpha \max \left\{p(x, y), p(y, T y) \frac{[1+p(x, T x)]}{1+p(x, y)}\right\}
$$

for all $x, y \in X$ where $\alpha \in(0,1)$. Then $T$ has a fixed point.

Proof. Let $x_{0} \in X$ and $x_{1} \in T x_{0}$. By using Lemma 2, we have $x_{2} \in T x_{1}$ such that

$$
p\left(x_{1}, x_{2}\right) \leq \frac{1}{\sqrt{\alpha}} H_{p}\left(T x_{0}, T x_{1}\right) .
$$

for $\frac{1}{\sqrt{\alpha}}>1$. From (1) and above inequality, we get

$$
p\left(x_{1}, x_{2}\right) \leq \frac{1}{\sqrt{\alpha}} \alpha \max \left\{p\left(x_{0}, x_{1}\right), p\left(x_{1}, T x_{1}\right) \frac{\left[1+p\left(x_{0}, T x_{0}\right)\right]}{1+p\left(x_{0}, x_{1}\right)}\right\} \leq \sqrt{\alpha} \max \left\{p\left(x_{0}, x_{1}\right), p\left(x_{1}, x_{2}\right)\right\} .
$$


Now, if $\max \left\{p\left(x_{0}, x_{1}\right), p\left(x_{1}, x_{2}\right)\right\}=p\left(x_{1}, x_{2}\right)$, then we get a contradiction. Thus, $\max \left\{p\left(x_{0}, x_{1}\right), p\left(x_{1}, x_{2}\right)\right\}=p\left(x_{0}, x_{1}\right)$. So, we have

$$
p\left(x_{1}, x_{2}\right) \sqrt{\alpha} p\left(x_{0}, x_{1}\right) .
$$

Again from (1), for $x_{2} \in T x_{1}$, there exists $x_{3} \in T x_{2}$ such that

$$
p\left(x_{2}, x_{3}\right) \leq \frac{1}{\sqrt{\alpha}} H_{p}\left(T x_{1}, T x_{2}\right)
$$

From (1) and above, we get

$$
p\left(x_{2}, x_{3}\right) \leq \frac{1}{\sqrt{\alpha}} \alpha \max \left\{p\left(x_{1}, x_{2}\right), p\left(x_{2}, T x_{2}\right) \frac{\left[1+p\left(x_{1}, T x_{1}\right)\right]}{1+p\left(x_{1}, x_{2}\right)}\right\} \leq \sqrt{\alpha} \max \left\{p\left(x_{1}, x_{2}\right), p\left(x_{2}, x_{3}\right)\right\} .
$$

Now, if $\max \left\{p\left(x_{1}, x_{2}\right), p\left(x_{2}, x_{3}\right)\right\}=p\left(x_{2}, x_{3}\right)$, then we get a contradiction. Thus, $\max \left\{p\left(x_{1}, x_{2}\right), p\left(x_{2}, x_{3}\right)\right\}=p\left(x_{1}, x_{2}\right)$. So, we have

$$
p\left(x_{2}, x_{3}\right) \sqrt{\alpha} p\left(x_{1}, x_{2}\right) .
$$

Continuing this process, we get $\left\{x_{n}\right\} \subset X$ and $x_{n-1} \in T x_{n}$ such that

$$
p\left(x_{n}, x_{n+1}\right) \leq \sqrt{\alpha} p\left(x_{n-1}, x_{n}\right) \text { for all } n \in \mathbb{N}
$$

From here we obtain $p\left(x_{n}, x_{n+1}\right) \leq(\sqrt{\alpha})^{n} p\left(x_{0}, x_{1}\right)$ for all $n \in \mathbb{N}$. Using this and the property (p4) of a partial metric, for any $m \in \mathbb{N}$, we have

$$
\begin{aligned}
p\left(x_{n}, x_{n+m}\right) & \leq p\left(x_{n}, x_{n+1}\right)+p\left(x_{n+1}, x_{n+2}\right)+\ldots+p\left(x_{n+m-1}, x_{n+m}\right) \\
& \leq(\sqrt{\alpha})^{n} p\left(x_{0}, x_{1}\right)+(\sqrt{\alpha})^{n+1} p\left(x_{0}, x_{1}\right)+\ldots+(\sqrt{\alpha})^{n+m-1} p\left(x_{0}, x_{1}\right) \\
& =\left((\sqrt{\alpha})^{n}+(\sqrt{\alpha})^{n+1}+\ldots+(\sqrt{\alpha})^{n+m-1}\right) p\left(x_{0}, x_{1}\right) \\
& \leq \frac{(\sqrt{\alpha})^{n}}{1-\sqrt{\alpha}} p\left(x_{0}, x_{1}\right) \rightarrow 0 \text { as } n \rightarrow+\infty
\end{aligned}
$$

since $0<\alpha<1$. This shows that $\left\{x_{n}\right\}$ is a Cauchy sequence in $\left(X, p^{s}\right)$. Since $(X, p)$ is a complete partial metric space, by Lemma $1,\left(X, p^{s}\right)$ is a complete metric space. Therefore, the sequence $\left\{x_{n}\right\}$ converges to some $x \in X$ with respect to the metric $p^{s}$, that is, $\lim _{n \rightarrow \infty} p^{s}\left(x_{n}, x\right)=0$. Again from Remark 1, we have

$$
p(x, x)=\lim _{n \rightarrow \infty} p\left(x_{n}, x\right)=\lim _{n, m \rightarrow \infty} p\left(x_{n}, x_{m}\right)=0 .
$$

Now, we show that $p(x, T x)=0$. On the contrary, suppose that $p(x, T x)>0$. By using the (p4) inequality, (1) and (2), we have

$$
\begin{aligned}
p(x, T x) & \leq p\left(x, x_{n+1}\right)+p\left(x_{n+1}, T x\right)-p\left(x_{n+1}, x_{n+1}\right) \\
& \leq p\left(x, x_{n+1}\right)+H_{p}\left(T x_{n}, T x\right) \\
& \leq p\left(x, x_{n+1}\right)+\alpha \max \left\{p\left(x_{n}, x\right), p(x, T x) \frac{1+p\left(x_{n}, T x_{n}\right)}{1+p\left(x_{n}, x\right)}\right\}
\end{aligned}
$$

letting $n \rightarrow \infty$, we get

$$
p(x, T x) \leq \alpha p(x, T x) .
$$


But, this is impossible for $\alpha<1$. Thus, $p(x, T x)=0$. Therefore, we have

$$
p(x, T x)=0=p(x, x)
$$

which from Remark 1 implies that $x \in T x$.

Example 2. Let $X=\{0,1,3,7\}$ and $p(x, y)=\max \{x, y\}$ for all $x, y \in X$. It is clear that $(X, p)$ is a complete partial metric space. Let $T: X \rightarrow \Omega_{C_{p}}(X)$ be defined by $T(0)=T(1)=\{0\}, T(3)=\{0,1\}$ and $T(7)=\{0,3\}$. It is easy that the subsets $\{0\},\{0,1\}$ and $\{0,3\}$ are bounded. So, if $x \in\{0,1,3,7\}$, then we get

$$
x \in \overline{\{0\}} \Leftrightarrow p(x,\{0\})=p(x, x) \Leftrightarrow p(x,\{0\})=x \Leftrightarrow x \in\{0\}
$$

Also,

$$
x \in \overline{\{0,1\}} \Leftrightarrow p(x,\{0,1\})=p(x, x) \Leftrightarrow \min \{p(x, 0), p(x, 1)\}=x \Leftrightarrow x \in\{0,1\}
$$

And,

$$
x \in \overline{\{0,3\}} \Leftrightarrow p(x,\{0,3\})=p(x, x) \Leftrightarrow \min \{p(x, 0), p(x, 3)\}=x \Leftrightarrow x \in\{0,3\} .
$$

Hence, $\{0\},\{0,1\}$ and $\{0,3\}$ are closed with respect to the partial metric $p$. Now, we will show that, for all $x, y \in X$, the contractive condition (1) is satisfied with $\alpha=\frac{1}{2}$. To see that it is sufficient to consider the following four cases:

If $x, y \in\{0,1\}$, then (1) trivially holds.

If $x \in\{0,1\}$ and $y=3$, then

$$
H_{p}(T x, T y)=1 \leq \alpha \max \left\{p(x, y), p(y, T y) \frac{1+p(x, T x)}{1+p(x, y)}\right\} .
$$

If $x \in\{0,1\}$ and $y=7$, then

$$
H_{p}(T x, T y)=3 \leq \alpha \max \left\{p(x, y), p(y, T y) \frac{1+p(x, T x)}{1+p(x, y)}\right\} .
$$

If $x, y \in\{3,7\}$, then

$$
H_{p}(T x, T y)=3 \leq \alpha \max \left\{p(x, y), p(y, T y) \frac{1+p(x, T x)}{1+p(x, y)}\right\} .
$$

Thus, all the conditions of Theorem 3 are satisfied and $x=0$ is a fixed point of $T$.

Now, we state another fixed point theorem on a complete partial metric space.

Theorem 4. Let $(X, p)$ be a complete partial metric space and let $T: X \rightarrow \Omega_{C}^{p}(X)$ be a set-valued map such that

$$
H_{p}(T x, T y) \leq \alpha\{p(x, T x) p(y, T y)\}^{\frac{1}{2}}
$$

for all $x, y \in X$ where $\alpha \in(0,1)$. Then $T$ has a fixed point.

Proof. Let $x_{0} \in X$ and $x_{1} \in T x_{0}$. By using Lemma 2, we have $x_{2} \in T x_{1}$ such that

$$
p\left(x_{1}, x_{2}\right) \leq \frac{1}{\sqrt{\alpha}} H_{p}\left(T x_{0}, T x_{1}\right) \text {. }
$$


for $\frac{1}{\sqrt{\alpha}}>1$. From (3) and (4), we obtain

$$
p\left(x_{1}, x_{2}\right) \leq \frac{1}{\sqrt{\alpha}} \alpha\left\{p\left(x_{0}, T x_{0}\right) p\left(x_{1}, T x_{1}\right)\right\}^{\frac{1}{2}} \leq \sqrt{\alpha} p\left(x_{0}, x_{1}\right)^{\frac{1}{2}} p\left(x_{1}, x_{2}\right)^{\frac{1}{2}} \leq \alpha p\left(x_{0}, x_{1}\right)
$$

Continuing the same way, we get $\left\{x_{n}\right\} \subset X$ and $x_{n-1} \in T x_{n}$ such that

$$
p\left(x_{n}, x_{n+1}\right) \leq \alpha p\left(x_{n-1}, x_{n}\right) \leq \alpha^{2} p\left(x_{n-2}, x_{n-1}\right) \leq \ldots \leq \alpha^{n} p\left(x_{0}, x_{1}\right) \text { for all } n \in \mathbb{N} .
$$

Using this and the property (p4) of a partial metric, for any $m \in \mathbb{N}$, we have

$$
\begin{aligned}
p\left(x_{n}, x_{n+m}\right) & \leq p\left(x_{n}, x_{n+1}\right)+p\left(x_{n+1}, x_{n+2}\right)+\ldots+p\left(x_{n+m-1}, x_{n+m}\right) \\
& \leq \alpha^{n} p\left(x_{0}, x_{1}\right)+\alpha^{n+1} p\left(x_{0}, x_{1}\right)+\ldots+\alpha^{n+m-1} p\left(x_{0}, x_{1}\right) \\
& =\left(\alpha^{n}+\alpha^{n+1}+\ldots+\alpha^{n+m-1}\right) p\left(x_{0}, x_{1}\right) \\
& \leq \frac{\alpha^{n}}{1-\alpha} p\left(x_{0}, x_{1}\right) \rightarrow 0 \text { as } n \rightarrow+\infty
\end{aligned}
$$

since $0<\alpha<1$. This shows that $\left\{x_{n}\right\}$ is a Cauchy sequence in $\left(X, p^{s}\right)$. Since $(X, p)$ is a complete partial metric space, by Lemma $1,\left(X, p^{s}\right)$ is a complete metric space. Therefore, the sequence $\left\{x_{n}\right\}$ converges to some $x \in X$ with respect to the metric $p^{s}$, that is, $\lim _{n \rightarrow \infty} p^{s}\left(x_{n}, x\right)=0$. Again from Remark 1, we have

$$
p(x, x)=\lim _{n \rightarrow \infty} p\left(x_{n}, x\right)=\lim _{n, m \rightarrow \infty} p\left(x_{n}, x_{m}\right)=0 .
$$

Now, we show that $p(x, T x)=0$. Assume this is not true the from (p4) inequality, (3) and (5), we have

$$
\begin{aligned}
p(x, T x) & \leq p\left(x, x_{n+1}\right)+p\left(x_{n+1}, T x\right)-p\left(x_{n+1}, x_{n+1}\right) \\
& \leq p\left(x, x_{n+1}\right)+H_{p}\left(T x_{n}, T x\right) \\
& \leq p\left(x, x_{n+1}\right)+\alpha\left\{p\left(x_{n}, T x_{n}\right) p(x, T x)\right\}^{\frac{1}{2}} .
\end{aligned}
$$

Passing to limit as $n \rightarrow \infty$, we get

$$
p(x, T x) \leq 0 .
$$

which is a contradiction. Thus, $p(x, T x)=0$. Thus, we get

$$
p(x, T x)=0=p(x, x)
$$

and $x \in T x$.

We can give the following another two fixed point theorems for set-valued mapping defined in complete partial metric spaces.

Theorem 5. Let $(X, p)$ be a complete partial metric space and let $T: X \rightarrow \Omega_{C}^{p}(X)$ be a set-valued map such that

$$
H_{p}(T x, T y) \leq \alpha\left\{\frac{p(x, T x) p(x, T y)+p(y, T y) p(y, T x)}{p(x, T y)+p(y, T y)}\right\}
$$

where $\alpha \in(0,1)$ and $p(x, T y)+p(y, T y) \neq 0$ for all $x, y \in X$. Then $T$ has a fixed point. 
Proof. Let $x_{0} \in X, x_{1} \in T x_{0}$ and $p(x, T y)+p(y, T y) \neq 0$. By using Lemma 2, we have $x_{2} \in T x_{1}$ such that

$$
p\left(x_{1}, x_{2}\right) \leq \frac{1}{\sqrt{\alpha}} H_{p}\left(T x_{0}, T x_{1}\right) .
$$

for $\frac{1}{\sqrt{\alpha}}>1$. From (6) and (7), we have

$$
\begin{aligned}
p\left(x_{1}, x_{2}\right) & \leq \frac{1}{\sqrt{\alpha}} \alpha\left\{\frac{p\left(x_{0}, T x_{0}\right) p\left(x_{0}, T x_{1}\right)+p\left(x_{1}, T x_{1}\right) p\left(x_{1}, T x_{0}\right)}{p\left(x_{0}, T x_{1}\right)+p\left(x_{1}, T x_{1}\right)}\right\} \\
& \leq \sqrt{\alpha}\left\{\frac{p\left(x_{0}, x_{1}\right) p\left(x_{0}, x_{2}\right)+p\left(x_{1}, x_{2}\right) p\left(x_{1}, x_{1}\right)}{p\left(x_{0}, x_{2}\right)+p\left(x_{1}, x_{2}\right)}\right\} \\
& \leq \sqrt{\alpha} p\left(x_{0}, x_{1}\right)
\end{aligned}
$$

Continuing this process, we obtain $\left\{x_{n}\right\} \subset X$ and $x_{n-1} \in T x_{n}$ such that

$$
p\left(x_{n}, x_{n+1}\right) \leq \sqrt{\alpha} p\left(x_{n-1}, x_{n}\right) \leq \sqrt{\alpha}^{2} p\left(x_{n-2}, x_{n-1}\right) \leq \ldots \leq \sqrt{\alpha}^{n} p\left(x_{0}, x_{1}\right) \text { for all } n \in \mathbb{N} .
$$

Using this and the property (p4) of a partial metric, for any $m \in \mathbb{N}$, we have

$$
\begin{aligned}
p\left(x_{n}, x_{n+m}\right) & \leq p\left(x_{n}, x_{n+1}\right)+p\left(x_{n+1}, x_{n+2}\right)+\ldots+p\left(x_{n+m-1}, x_{n+m}\right) \\
& \leq \sqrt{\alpha}^{n} p\left(x_{0}, x_{1}\right)+\sqrt{\alpha}^{n+1} p\left(x_{0}, x_{1}\right)+\ldots+\sqrt{\alpha}^{n+m-1} p\left(x_{0}, x_{1}\right) \\
& \leq \frac{\sqrt{\alpha}^{n}}{1-\sqrt{\alpha}} p\left(x_{0}, x_{1}\right) \rightarrow 0 \text { as } n \rightarrow+\infty
\end{aligned}
$$

since $0<\alpha<1$. This yields that $\left\{x_{n}\right\}$ is a Cauchy sequence in $\left(X, p^{s}\right)$. Since $(X, p)$ is a complete partial metric space, by Lemma $1,\left(X, p^{s}\right)$ is a complete metric space. Therefore, the sequence $\left\{x_{n}\right\}$ converges to some $x \in X$ with respect to the metric $p^{s}$, that is, $\lim _{n \rightarrow \infty} p^{s}\left(x_{n}, x\right)=0$. Again from Remark 1, we have

$$
p(x, x)=\lim _{n \rightarrow \infty} p\left(x_{n}, x\right)=\lim _{n, m \rightarrow \infty} p\left(x_{n}, x_{m}\right)=0 .
$$

From (p4) inequality and (6), we have

$$
\begin{aligned}
p(x, T x) & \leq p\left(x, x_{n+1}\right)+p\left(x_{n+1}, T x\right)-p\left(x_{n+1}, x_{n+1}\right) \\
& \leq p\left(x, x_{n+1}\right)+H_{p}\left(T x_{n}, T x\right) \\
& \leq p\left(x, x_{n+1}\right)+\alpha\left\{\frac{p\left(x_{n}, T x_{n}\right) p\left(x_{n}, T x\right)+p\left(x_{n}, T x_{n}\right) p\left(x, T x_{n}\right)}{p\left(x_{n}, T x\right)+p(x, T x)}\right\} .
\end{aligned}
$$

Letting $n \rightarrow \infty$, we get

$$
p(x, T x) \leq 0
$$

But, this is impossible. Thus, $p(x, T x)=0$. Therefore, we get

$$
p(x, T x)=0=p(x, x)
$$

which from Remark 1 implies that $x \in T x$. 
Theorem 6. Let $(X, p)$ be a complete partial metric space and let $T: X \rightarrow \Omega_{C}^{p}(X)$ be a set-valued map such that

$$
H_{p}(T x, T y) \leq \alpha\left\{\frac{p(x, T x) p(x, T y)+[p(x, y)]^{2}+p(x, T x) p(x, y)}{p(x, T x)+p(x, y)+p(x, T y)}\right\}
$$

where $\alpha \in(0,1)$ and $p(x, T x)+p(x, y)+p(x, T y) \neq 0$ for all $x, y \in X$. Then $T$ has a fixed point.

Proof. Let $x_{0} \in X, x_{1} \in T x_{0}$ and $p(x, T x)+p(x, y)+p(x, T y) \neq 0$. By using Lemma 2, we have $x_{2} \in T x_{1}$ such that

$$
p\left(x_{1}, x_{2}\right) \leq \frac{1}{\sqrt{\alpha}} H_{p}\left(T x_{0}, T x_{1}\right)
$$

for $\frac{1}{\sqrt{\alpha}}>1$. From (8) and (9), we obtain

$$
\begin{aligned}
p\left(x_{1}, x_{2}\right) & \leq \frac{1}{\sqrt{\alpha}} \alpha\left\{\frac{p\left(x_{0}, T x_{0}\right) p\left(x_{0}, T x_{1}\right)+\left[p\left(x_{0}, x_{1}\right)\right]^{2}+p\left(x_{0}, T x_{0}\right) p\left(x_{0}, x_{1}\right)}{p\left(x_{0}, T x_{0}\right)+p\left(x_{0}, x_{1}\right)+p\left(x_{0}, T x_{1}\right)}\right\} \\
& \leq \sqrt{\alpha}\left\{\frac{p\left(x_{0}, x_{1}\right) p\left(x_{0}, x_{2}\right)+\left[p\left(x_{0}, x_{1}\right)\right]^{2}+p\left(x_{0}, x_{1}\right) p\left(x_{0}, x_{1}\right)}{2 p\left(x_{0}, x_{1}\right)+p\left(x_{0}, x_{2}\right)}\right\} \\
& \leq \sqrt{\alpha} p\left(x_{0}, x_{1}\right) .
\end{aligned}
$$

On repeating this process, we get $\left\{x_{n}\right\} \subset X$ and $x_{n-1} \in T x_{n}$ such that

$$
p\left(x_{n}, x_{n+1}\right) \leq \sqrt{\alpha} p\left(x_{n-1}, x_{n}\right) \leq \sqrt{\alpha}^{2} p\left(x_{n-2}, x_{n-1}\right) \leq \ldots \leq \sqrt{\alpha}^{n} p\left(x_{0}, x_{1}\right) \text { for all } n \in \mathbb{N} .
$$

Using this and the property (p4) of a partial metric, for any $m \in \mathbb{N}$, we have

$$
\begin{aligned}
p\left(x_{n}, x_{n+m}\right) & \leq p\left(x_{n}, x_{n+1}\right)+p\left(x_{n+1}, x_{n+2}\right)+\ldots+p\left(x_{n+m-1}, x_{n+m}\right) \\
& \leq \sqrt{\alpha}^{n} p\left(x_{0}, x_{1}\right)+\sqrt{\alpha}^{n+1} p\left(x_{0}, x_{1}\right)+\ldots+\sqrt{\alpha}^{n+m-1} p\left(x_{0}, x_{1}\right) \\
& \leq \frac{\sqrt{\alpha}^{n}}{1-\sqrt{\alpha}^{\prime}} p\left(x_{0}, x_{1}\right) \rightarrow 0 \text { as } n \rightarrow+\infty
\end{aligned}
$$

since $0<\alpha<1$. This shows that $\left\{x_{n}\right\}$ is a Cauchy sequence in $\left(X, p^{s}\right)$. Since $(X, p)$ is a complete partial metric space, by Lemma $1,\left(X, p^{s}\right)$ is a complete metric space. Therefore, the sequence $\left\{x_{n}\right\}$ converges to some $x \in X$ with respect to the metric $p^{s}$, that is, $\lim _{n \rightarrow \infty} p^{s}\left(x_{n}, x\right)=0$. From Remark 1 , we have

$$
p(x, x)=\lim _{n \rightarrow \infty} p\left(x_{n}, x\right)=\lim _{n, m \rightarrow \infty} p\left(x_{n}, x_{m}\right)=0 .
$$

Now, we show that $p(x, T x)=0$. Now, we shall show that $x$ is a fixed point of $T$. For any $n \in \mathbb{N}$ it follows from (p4) and (7) that

$$
\begin{aligned}
p(x, T x) & \leq p\left(x, x_{n+1}\right)+p\left(x_{n+1}, T x\right)-p\left(x_{n+1}, x_{n+1}\right) \\
& \leq p\left(x, x_{n+1}\right)+H_{p}\left(T x_{n}, T x\right) \\
& \leq p\left(x, x_{n+1}\right)+\alpha \frac{p\left(x_{n}, T x_{n}\right) p\left(x_{n}, T x\right)+\left[p\left(x_{n}, x\right)\right]^{2}+p\left(x_{n}, T x_{n}\right) p\left(x_{n}, x\right)}{p\left(x_{n}, T x_{n}\right)+p\left(x_{n}, x\right)+p\left(x_{n}, T x\right)} .
\end{aligned}
$$

Letting $n \rightarrow \infty$, we get

$$
p(x, T x) \leq 0 .
$$


which is a contraction. That is, $p(x, T x)=0$. Thus, we obtain

$$
p(x, T x)=0=p(x, x)
$$

which from Remark 1 implies that $x \in T x$.

\section{Conclusions}

In this study, we investigate some contraction mapping in partial Hausdorff metric space and prove the existence of fixed point of this mapping in partial Hausdorff metric. Further, we also give an example in support our result.

\section{Competing interests}

The authors declare that they have no competing interests.

\section{Authors' contributions}

All authors have contributed to all parts of the article. All authors read and approved the final manuscript.

\section{References}

[1] S. G. Matthews, Partial metric topology, In: Proc. 8th Summer Conference on General Topology and Applications, in: Ann. New York Acad. Sci., vol. 728, 1994, pp. 183-197.

[2] E. Karapınar, S. Romaguera, Nonunique fixed point theorems in partial metric spaces, Filomat, 27 (7), 2013, 1305-1314.

[3] E. Karapınar, W. Shatanawi, K. Taş, Fixed Point theorem on partial metric spaces involving rational expressions, Miskolc Math. Notes, 14, 2013, 135-142.

[4] I. Altun, H. Simsek, Some fixed point theorems on dualistic partial metric spaces, J. Adv. Math. Stud., 1, 2008, 1-8.

[5] I. Altun, F. Sola, H. Simsek, Generalized contractions on partial metric spaces, Topology Appl., 157, 2010, 2778-2785.

[6] J. Jachymski, The contraction principle for mappings on a metric space with a graph, Proc. Am. Math. Soc., 136 (4), 2008 , 1359-1373.

[7] H. Aydi, M. Abbas, C. Vetro, Partial Hausdorff metric and Nadler's fixed point theorem on partial metric spaces, Topol. Appl., 159, 2012, 3234-3242.

[8] H. Aydi, M. Abbas, C. Vetro, Common fixed points for multivalued generalized contractions on partial metric spaces, RACSAMRevista de la Real Academia de Ciencias Exactas, Fisicas y Naturales. Serie A. Mathematicas, 108, 2014, 483-501.

[9] H. Aydi, E. Karapınar, C. Vetro, On Ekeland's variational principle in partial metric spaces, Appl. Math. Inf. Sci., 9, No.1, 2015, 257-262.

[10] R. H. Haghi, S. Rezapour, N. Shahzad, Be careful on partial metric fixed point results, Topol. Appl., 160, 2013, 450-454.

[11] S. Oltra, O. Valero, Banach's fixed point theorem for partial metric spaces, Rend. Istit. Mat. Univ. Trieste, 36, $2004,17-26$.

[12] S. Romaguera, A Kirk type characterization of completeness for partial metric spaces, Fixed Point Theory Appl., 2010, Article ID 493298, 6pp.

[13] On Banach fixed point theorems for partial metric spaces, Appl. Gen. Topol., 6 (2), 2005, 229-240.

[14] S. B. Nadler, Multivalued contraction mappings, Pacific J. Math., 30 1969, 475-488.

[15] C. Vetro, F Vetro, Metric or partial metric spaces endowed with a finite number of graphs: a tool to obtain fixed point results, Topol. Appl., 164, 2014, 125-137.

[16] M. Samreen, T. Kamran, Fixed Point Theorems for integral G-contractions, Fixed Point Theory Appl., 149, 2013, doi:10.1186/1687-1812-2013-149. 
[17] T. Kamran, M. Samreen, N. Shahzad, Probabilistic G-contractions, Fixed Point Theory Appl., 223, 2013, doi:10.1186/1687-18122013-223.

[18] F. Bajor, Fixed point theorems for Reich type contractions on metric spaces with a graph, Nonlinear anal., 75, $2012,3895-3901$. 